\title{
Factores de riesgo para el consumo de alcohol en adolescentes estudiantes
}

\author{
Graciela Arrioja Morales ${ }^{1}$ \\ Sueli Aparecida Frari Galera² \\ Alejandro Torres Reyes ${ }^{1}$ \\ Sebastiana del Rosario Gargantúa Aguila ${ }^{1}$ \\ María Luz de Avila Arroyo ${ }^{1}$ \\ Francisco Adrian Morales Castillo ${ }^{1}$
}

Introducción: Las legislaciones de los países no aceptan el consumo de alcohol en adolescentes, pero se observa incremento en ellos. Objetivo: Identificar el efecto de los factores personales en el consumo de alcohol en adolescentes que estudian. Metodos: diseño correlacional en adolescentes que estudian, con muestreo aleatorio se selecciona una $n=894$, se aplican tres instrumentos. Resultados: El consumo de alcohol alguna vez en la vida fue del $65 \%$. Se encuentran efectos de los factores de riesgo; sexo y edad sobre el consumo de alcohol ( $c 2=31.23 ; \mathrm{p}=.001 ; \mathrm{R} 2=27 \%$ ). Conclusiones: Los factores de riesgo incrementan el consumo de alcohol en adolescentes.

Descriptores: Factores de Riesgo; Alcoholismo; Enfermería.

\footnotetext{
${ }^{1}$ MSc, Professor, Facultad de Enfermería, Benemérita Universidad Autónoma de Puebla, Puebla, Mexico.

${ }^{2} \mathrm{PhD}$, Associate Professor, Escola de Enfermagem de Ribeirão Preto, Universidade de São Paulo, WHO Collaborating Centre for Nursing Research Development, Ribeirão Preto, SP, Brazil.
}

\author{
Correspondencia: \\ Graciela Arrioja Morales \\ Benemérita Universidad Autónoma de Puebla; Facultad de Enfermería \\ 27 poniente 1304 \\ Bairro: Colonia Volcanes \\ CEP: 72410, Puebla, Pue, Mexico \\ E-mail: garrioja@yahoo.com
}




\section{Fatores de risco para o consumo de álcool em adolescentes estudantes}

Introdução: As legislações dos países não aceitam o consumo de álcool em adolescentes, mas se observa seu aumento. Objetivo: Identificar o efeito dos fatores pessoais no consumo de álcool em adolescentes que estudam. Métodos: Estudo correlacional em adolescentes que estudam, com amostragem aleatória realizando a seleção de uma $n=894$, a aplicação de três instrumentos. Resultados: O consumo de álcool alguma vez na vida foi de $65 \%$. Encontram-se efeitos dos fatores de risco; sexo e idade sobre o consumo de álcool (c2=31.23; $p=.001 ; R 2=27 \%$ ). Conclusões: Os fatores de risco aumentam o consumo de álcool em adolescentes.

Descritores: Fatores de Risco; Alcoolismo; Enfermagem.

\section{Risk factors for alcohol consumption in adolescents students}

Introduction: Countries' regulations do not accept alcohol consumption in adolescents, but it has been increasing. Objective: To identify the effect of personal factors in alcohol consumption in studying adolescents Methods: this is a correlational design in studying adolescents with random sampling, if there is $n=894$ three instruments is apply. Results: At least once in life alcohol consumption was $65 \%$. Effects of risk factors: gender and age on alcohol consumption are $(c 2=31.23 ; p=.001 ; R 2=27 \%)$. Final remarks: Risk factors step up alcohol consumption in adolescents.

Descriptors: Risk Factors; Alcoholism; Nursing.

\section{Introducción}

En el mundo durante 2010 una de cada 20 personas consumió drogas ilícitas y el equivalente a 230 millones de personas son consumidoras de alcohol principalmente en edades tempranas. El alcohol es la droga más consumida por adolescentes, dado la alta diversidad y formas de distribución de bebidas alcohólicas a adolescentes de todos los estratos sociales $^{(1-3)}$. El fenómeno del consumo de alcohol, es complejo y multicausal de impacto sociopolítico, económico y psicossocial en todos los países ${ }^{(4-6)}$. El consumo de alcohol se encuentra entre los cinco primeros factores de riesgo de accidentes ${ }^{(1,7-9)}$. Los adolescente tienen 4.4 veces más probabilidad de consumir alcohol si su padre consume; 4.6 veces más, si es el hermano y 10.4 veces si su mejor amigo consume. Si viven en familia tienen menos riesgo a consumir drogas ${ }^{(10)}$.

En México, el estado de Puebla ocupa el lugar 11 por encima de la media nacional en el consumo de bebidas alcohólicas, $7 \%$ de la población ( 7.800 casos), $47 \%$ de los hombres y el $15 \%$ de mujeres ${ }^{(11)}$.

La adolescencia varía por las características individuales o de grupo y comprende cambios anatomo-fisiológicos que modifican el perfil psicológico y personalidad. Son dos etapas: a) adolescencia temprana 10 a 14 años; b) adolescencia tardía 15 a 19 años. Los adolescentes hacen ajustes o modificaciones a medida en que se sienten aceptados socialmente. Su inestabilidad emocional por desconocimiento y temor a nuevas vivencias o toma de decisiones y baja autoestima lo pueden inducir al consumo de alcohol y consumo de drogas ilegales, problemas escolares, relaciones sexuales sin protección, problemas legales, alteraciones afectivas, accidentes de tráfico, suicidios y homicidios ${ }^{(12)}$.

La conducta del consumo de alcohol en los adolescentes se explica como resultado de las influencias de factores de riesgo, que pueden ser factores personales y su estudio merece más investigaciones científicas ${ }^{(13)}$ 
Con el estudio se continúa abordando el fenómeno del consumo de alcohol en los adolescentes mediante el planteamiento de la siguiente pregunta de investigación: ¿Cuál es el efecto de factores (personales y de riesgos) en el consumo de alcohol en adolescentes que estudian en escuelas secundarias del sistema educativo del estado de Puebla, México?

\section{Método}

\section{El diseño del estudio es correlacional}

Estudio correlacional, realizado en el ano de 2012 con adolescentes estudiantes hombres y mujeres, de 11 a 16 años de edad; de turno matutino, de los tres grados de escuelas secundarias públicas urbanas del sistema educativo del estado de Puebla, México. Muestreo aleatorio, nivel de confianza de 95\%, significancia .05 y una potencia de .90 , para una muestra $(n=894)$. La selección de las escuelas, fue aleatoria, la selección de participantes fue estratificada por asignación proporcional al tamaño de cada estrato. Los criterios de inclusión fueron estudiantes de secundaria matriculados con consentimiento libre y esclarecido firmado por el padre o tutor y el asentimiento firmado por el estudiante.

Tres instrumentos fueron aplicados, la cédula de datos personales (CDP), el historial de uso y dependencia de drogas (HUDD) y el cuestionario de tamizaje de problemas en adolescentes, (Problem Oriented Screening Instrument for Teenager [POSIT]), traducido y validado por Marino, et al. (1998) ${ }^{(14)}$. Los datos se trasformaron en índices de 0 al 100, es por eso que la mayor puntuación es el factor de riesgo. Para seleccionar la muestra se obtuvo el marco muestral de los estudiantes. Los datos se recogieron en las instituciones educativas seleccionadas en el año de 2012.

A lo dispuesto en la Ley General de Salud en materia de investigación (1987), se respetó la dignidad humana, se protegiron los derechos y bienestar de los participantes.

\section{Procedimiento}

Para el análisis de datos se utilizó el paquete estadístico Statistical Package for Social Science (SPSS) versión 18.0 para Windows para la estadística descriptiva e inferencial. Se obtuvó la confiabilidad del POSIT mediante el alfa de Cronbach arriba de .7000 .

La presente investigación tuvo la autorización de la comisión de ética e investigación de la Facultad de Enfermería de la Benemérita Universidad Autónoma de Puebla (FE-BUAP) con el registro P-2012-0044CIP y de la Secretaria de Educación Pública.

\section{Resultados}

Los hallazgos del estudio, se presentan de acuerdo al logro del objetivo de investigación en tres ítems: a) confiabilidad del instrumentos; b) estadística descriptiva; c) estadística inferencial.

Consistencia interna del instrumento POSIT o cuestionario de tamizaje de problemas en adolescentes, (Problem Oriented Screening Instrument for Teenager (POSIT), obtuvo consistencia interna aceptable de .8300 .

Estadística descriptiva: los factores personales (FP) biológicos, las mujeres estuvieron representadas por el $55 \%$, la edad entre 11 y 13 años obtuvó el $51 \%$. En los FP socioculturales, el $42 \%$ cursaban el tercer grado de secundaria. El $59 \%$ rara vez falta a clase, el $88 \%$ estudia solamente, el $38 \%$ trabaja en comercio.

Prevalencia global, lápsica y actual: la prevalencia global, lápsica y actual del consumo de alcohol; El consumo de alcohol alguna vez en la vida o prevalencia global fue de 65\% (IC95\%; 62-68\%). La prevalencia lápsica de alcohol de 31\% (IC95\%; 28 - 34\%). La prevalencia actual de alcohol de 20\% (IC95\%; 18 $23 \%$ ). Se encontraron diferencias significativas del consumo de alcohol por edad; la mayor proporción del consumo de alcohol alguna vez en la vida, en el último año y en el último mes fue en los adolescentes de 14 y 16 años.

Tabla 1 - Consumo de alcohol: comparación por género entre estudiantes-Puebla/México

\begin{tabular}{|c|c|c|c|c|c|c|c|c|c|c|c|c|}
\hline \multirow{3}{*}{ Variable } & \multicolumn{4}{|c|}{ Alguna vez en la vida } & \multicolumn{4}{|c|}{ En el último año } & \multicolumn{4}{|c|}{ En el último mes } \\
\hline & \multicolumn{2}{|c|}{ Sí } & \multicolumn{2}{|c|}{ No } & \multicolumn{2}{|c|}{ Sí } & \multicolumn{2}{|c|}{ No } & \multicolumn{2}{|c|}{$\mathrm{Si}$} & \multicolumn{2}{|c|}{ No } \\
\hline & $f$ & $\%$ & $f$ & $\%$ & $f$ & $\%$ & $f$ & $\%$ & $f$ & $\%$ & $f$ & $\%$ \\
\hline Masculino & 263 & 45 & 140 & 45 & 143 & 35 & 260 & 64 & 77 & 42 & 326 & 46 \\
\hline \multirow[t]{2}{*}{ Femenino } & 319 & 55 & 172 & 55 & 136 & 28 & 355 & 72 & 105 & 58 & 386 & 54 \\
\hline & \multicolumn{4}{|c|}{$X^{2}=.008, p=.928$} & \multicolumn{4}{|c|}{$X^{2}=6.249, p=.012$} & \multicolumn{4}{|c|}{$X^{2}=.709, p=.400$} \\
\hline
\end{tabular}

Fuente: CDP, Historial de Consumo de Drogas 
En la Tabla 1, se muestra que existe diferencia por sexo en el consumo de alcohol en el último año; en los demás consumos se observó en las mujeres proporciones de consumo ligeramente superiores a la de los hombres. En el consumo de alcohol comparado por grado escolar, existen diferencias estadísticamente significativas, del consumo de alcohol alguna vez en la vida $\left(\chi^{2}=49.569, p<.001\right)$, en el último año $\left(\chi^{2}=31.180, p<.001\right)$ y en el último mes $\left(\chi^{2}=41.285, p<.001\right)$; se destaca mayor prevalencia de consumo de alcohol en los adolescentes de segundo grado. Existen diferencias estadísticamente significativas del consumo de alcohol por ocupación; en el consumo de alguna vez en la vida $\left(\chi^{2}=17.935\right.$, $p=.001)$, en el último año $\left(\chi^{2}=14.771, p=.001\right)$ y en el último mes $\left(\chi^{2}=18.491, p=.001\right)$.

\section{Patrón de Consumo de Alcohol en Adolescentes que Estudian en Secundaria}

En un día típico que el patrón de consumo de bebidas alcohólicas en promedio es de 2 bebidas alcohólicas. La bebida preferida en un día típico, es la cerveza (35\%) seguida con mucha diferencia por el vodka $(7.3 \%)$ y la sidra con el $6.2 \%$. La edad promedio de inicio en el consumo de alcohol fue a la edad de 12.2 con una DE de1.93.

Con los resultados obtenidos se alcanzó el segundo objetivo.

Factores de riesgo frente al consumo de drogas: descriptivos y prueba de Kolmogorov- Smirnov.

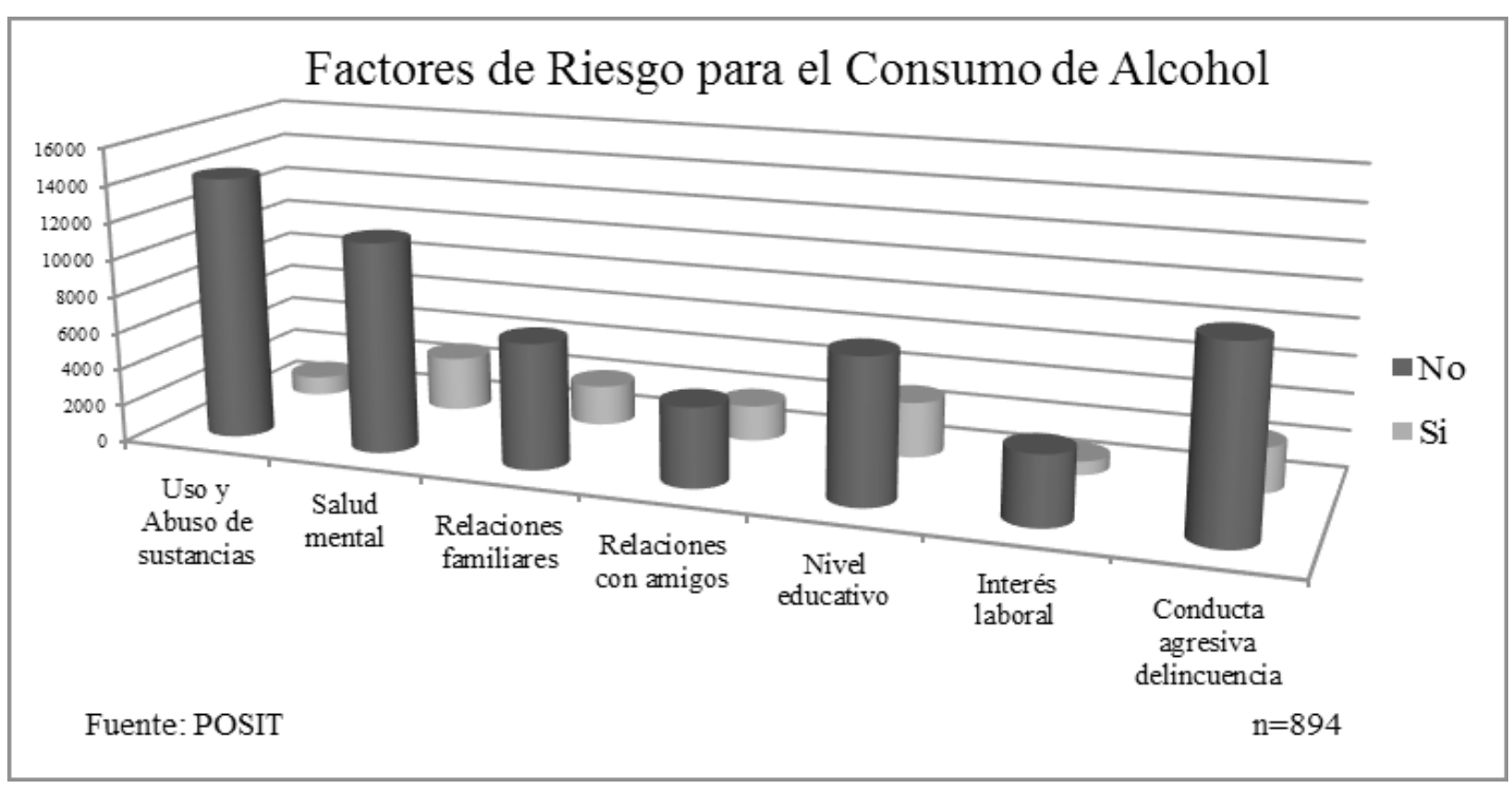

Figura 1 - medias de factores de riesgo (POSIT) en el consumo de alcohol.

Se econtraron medias de los factores de riesgo POSIT en consumo de alcohol son, para abuso de substancias $(\bar{X}=93.65, D E=64.32)$; interés laboral $(\bar{X}=85.71, D E=18.36)$; conducta agresiva $(\bar{X}=80.65$, $\mathrm{DE}=18.36) ; \mathrm{y}$ salud mental $(\bar{X}=79.50, \mathrm{DE}=21.27)$; relaciones familiares $(\bar{X}=40.54, D E=16.99)$, como se observa en la figura 1 , que los estudiantes viven en alto riego de involucrarse en el consumo de alcohol.

La prueba de Kolmogorov-Smirnov no indicó distribución normal para los datos del POSIT (K-S = 5.10 a 14.52: $p=.001$ ). Por lo que se decide utilizar estadística inferencial no paramétrica.
Descripción del instrumento POSIT por subescalas; para identificar los factores de riesgo (FR) frente al consumo de alcohol; por cada pregunta del POSIT se obtuvieron sus frecuencias para identificar las de mayor puntuación.

El factor de riesgo (FR), uso y abuso de sustancias por estudiantes de secundaria con 16 preguntas, el $70 \%$ obtuvieron una puntuación mayor al $90 \%$ del no uso y abuso de drogas. 


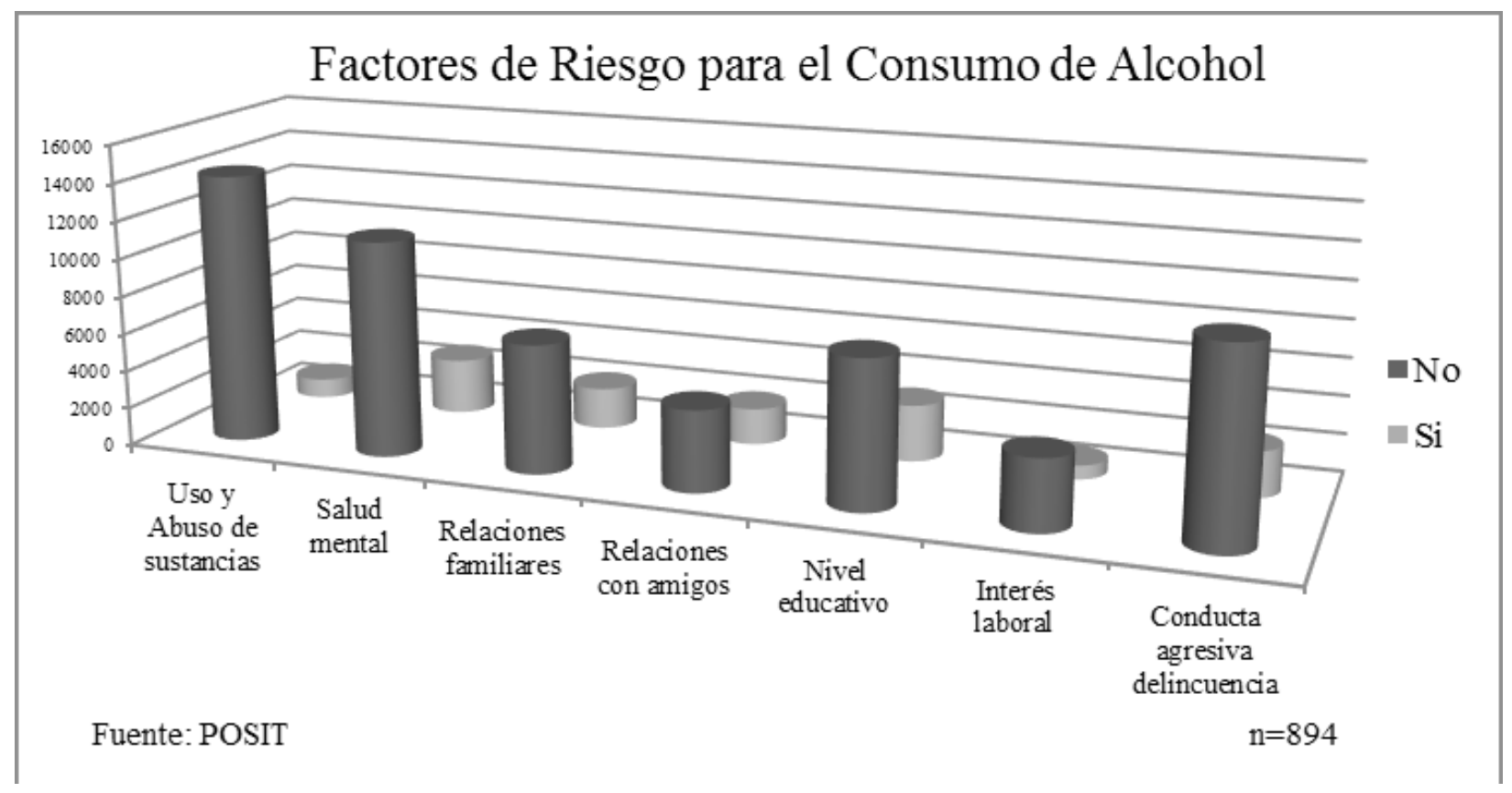

Figura 2 - Factores de riesgo y consumo de alcohol en adolescentes que estudian secundaria.

El FR, salud mental de los estudiantes de secundaria tiene 16 preguntas más del $22 \%$ refirio alguna dificultad. El FR, relaciones familiares con 10 preguntas más de $30 \%$ los estudiantes refirieron no tener buenas relaciones. El FR, relaciones con amigos con 10 preguntas, se encontró que más del $20 \%$ viven en este riesgo. El FR, nivel educativo con 12 preguntas, se encontró que más del $20 \%$ viven en este factor. EI FR, interés laboral de los estudiantes de secundaria tiene cinco preguntas el $11 \%$ refirió problemas. EI FR, conducta agresiva/delincuencia el $51 \%$ indicó tener por lo menos un indicador de riego.
Diferencia de factores de riesgo por consumo alguna vez en la vida, en el último año y en el último mes

Existe diferencia estadísticamente significativa de los FR con respecto al consumo de alcohol alguna vez en la vida, en el último año y en el último mes $(p<.001)$. Los resultados muestran medias más altas de FR en los adolescentes que consumen alcohol, en comparación de aquellos que no presentan consumo de esta sustancia (Tabla 2).

Tabla 2- Factores de riesgo y consumo de alcohol entre estudiantes-Puebla/México

\begin{tabular}{|c|c|c|c|c|c|c|}
\hline & Variable & Categoría & $n$ & $\bar{x}$ & U de Mann-Whitney & Valor de $p$ \\
\hline \multirow{7}{*}{$\begin{array}{l}\overline{0} \\
\frac{c}{0} \\
\frac{U}{4}\end{array}$} & Consumo alguna vez en la & $\mathrm{Si}$ & 582 & 503.01 & \multirow{2}{*}{58484.50} & \multirow{2}{*}{.001} \\
\hline & & No & 312 & 343.95 & & \\
\hline & Consumo en el último año & Si & 279 & 553.07 & \multirow{2}{*}{56337.50} & \multirow{2}{*}{.001} \\
\hline & & No & 615 & 399.61 & & \\
\hline & Consumo en el último mes & $\mathrm{Si}$ & 182 & 600.21 & \multirow{3}{*}{36999.50} & \multirow{3}{*}{.001} \\
\hline & & No & 712 & 408.47 & & \\
\hline & & No & 785 & 420.03 & & \\
\hline
\end{tabular}

\section{$\mathrm{n}=894$}

Fuente: POSIT, Historial del consumo de drogas psicoactivas

En la matriz de correlación de FR y consumo de alcohol, reportó relaciones débiles y moderadas; pero positivas y significativas: El consumo de alcohol alguna vez en la vida se relaciona con el POSIT ( $r=.294 ; p=.001$ ); con los FR; uso y abuso de sustancias $(r=.344 ; p=.001)$; con salud mental $(r=.167 ; p=.001)$, con relaciones familiares $(r=.137$; $p=.001)$, relaciones con amigos $(r=.226 ; p=.001)$, con nivel educativo $(r=.124 ; p=.001)$, interés laboral $(r=.137 ; p=.001)$, con conducta agresiva/delincuencia $(r=.331 ; p=.001)$. El consumo de alcohol en el último año se relaciona con el POSIT ( $r=.276 ; p=.001)$; con el uso y abuso de sustancias ( $r=.328 ; p=.001)$; con salud mental $(r=.170 ; p=.001)$, con relaciones familiares 
$(r=.152 ; p=.001)$, relaciones con amigos $(r=.170$; $p=.001)$, con nivel educativo $(r=.115 ; p=.001)$, interés laboral ( $r=.143 ; p=.001)$, con conducta agresiva/ delincuencia $(r=.292 ; p=.001)$. El consumo de alcohol en el último mes se relaciona con el POSIT ( $r=.299$; $p=.001)$; con uso y abuso de sustancias $(r=.410$; $p=.001) ;$ con salud mental $(r=.218 ; p=.001)$, con relaciones familiares $(r=.178 ; p=.001)$, relaciones con amigos $(r=.195 ; p=.001)$, con nivel educativo $(r=.105$; $p=.001)$, interés laboral $(r=.134 ; p=.001)$, con conducta agresiva/delincuencia $(r=.295 ; p=.001)$.
Efectos de los Factores de Riesgo sobre el Consumo de Drogas de los Adolescentes que Estudian en Secundaria.

Para determinar los efectos de los factores de riesgo (FR) sobre el consumo de alcohol, se construyeron 16 modelos de regresión logística. Las variables independientes fueron el géner, edad y FR, y las dependientes fueron los consumos de alcohol alguna vez en la vida, en el último año y en el último mes.

Tabla 3 - Modelos de regresión logística factores de riesgo, género y edad en el consumo de alcohol: alguna vez en la vida, en el último año y en el último mes

\begin{tabular}{lccccc}
\hline Consumo & Variable dependiente & MRL & $\mathrm{X}^{2}$ & Valor $\mathrm{p}$ & $\mathrm{R}^{2}$ \\
\hline \multirow{3}{*}{ Alcohol } & Alguna vez en la vida & 1 & 143.61 & .001 & $20 \%$ \\
& Último año & 2 & 121.80 & .001 & $18 \%$ \\
& Último mes & 3 & 107.44 & .001 & $18 \%$ \\
\hline
\end{tabular}

$\mathrm{n}=894$

Fuente: CDP, POSIT y HCD

En la Tabla 3 se presenta el modelo 1 de regresión logística $(M R L) 1$, en donde las variables independientes sexo, edad y los FR (abuso de sustancias, problemas de salud mental, relación con familiares, relación con amigo, bajo nivel educativo, interés laboral y conducta agresiva) afectan al consumo de alcohol alguna vez en la vida. Siendo la edad $(W=16.02 ; p=.001)$ impulsa el efecto. El MRL 2, muestra que las variables independientes sexo, edad y FR (abuso de sustancias, problemas de salud mental, relación con familiares, relación con amigos, bajo nivel educativo, interés laboral y conducta agresiva) influyen en el consumo de alcohol en el último año, efecto impulsado por el género $(W=4.27$; $p=.038)$ y la edad $(W=9.56 ; p=.003)$. En el MRL 3, las variables independientes sexo, edad y FR (abuso de sustancias, problemas de salud mental, relación con familiares, relación con amigos, bajo nivel educativo, interés laboral y conducta agresiva), influyen en el consumo de alcohol en el último mes, el efecto lo impulsa la edad $(\mathrm{W}=12.40 ; p=.001)$.

\section{Discusión}

Los hallazgos encontrados en los factores personales biológicos, socioculturales, diferen a lo reportado por Molinero, Salguero, Castro, Mora y Márquez, (2011) y a Gómez, et al., (2006)(15-16).

En cuanto a la prevalencia global "consumo de alcohol alguna vez en la vida" fue menor a lo reportado (80\%) por Gómez, et. al. (2006) ${ }^{(16)}$; Orgaz, Segovia, López \& Tricio $(2005)^{(17)}$ y a la reportada $(71 \%)$ por el Consejo Nacional Contra las Adicciones (CONADIC) en 2008(18). Apoya los hallazgos de la Comisión Nacional de Investigación en Drogas $(2001)^{(19)}$; UNODC (2006) $)^{(1)}$. La prevalencia lápsica (31\%) "consumo de alcohol en el último año, menor a la reportada (52\%) por el CONADIC $(2008)^{(18)}$. La prevalencia actual o en los "últimos 30 días" es de $20 \%$, menor a la presentada $(62 \%)$ por Gómez, et. al. (2006) $)^{(16)}$ y a la reportada $(41 \%)$ por CONADIC $(2008)^{(18)}$.

$\mathrm{Se}$ encuentran diferencias significativas del consumo de alcohol por edad y género; muy parecido a los resultados de Molinero, Salguero, Castro, Mora, y Márquez, (2011) ${ }^{(15)}$.

La bebida preferida por los estudiantes de secundaría fue similar a los reportados por Herrera, Wagner, Velazco, Borges \& Lazcano (2004)(8); Gómez, et al $(2006)^{(16)}$ y por lo mostrado a nivel nacional por el CONADIC $(2008)^{(18)}$ y INSP, $(2008)^{(20)}$. La edad promedia en años como de inicio en el consumo de alcohol fue similar a lo presentado por otros investigadores: (Herrera, Wagner, Velazco, Borges \& Lazcano, 2004) $)^{(8)}$; Gómez et al., 2006 ${ }^{(16)}$ y organismos a nivel nacional CONADIC (2008)(18) y INSP (2008) (20). La frecuencia con la que consumen alcohol en los últimos 30 días fue similar a lo que sucede a nivel nacional (CONADIC, 2008)(18) y INSP $(2008)^{(20)}$.

Los hallazgos en el que se identificó a los factores de riesgo (FR) frente al consumo de alcohol en adolescentes, son consistentes a lo planteado por Anicama, (2001), Clayton (1992); Nazar et al. (1994), Hawkins, Catalano \& Miller (1992) $)^{(21-24)}$, sobre 
todo en la salud mental, conducta agresiva, nivel de educación y relaciones familiares son condiciones y características individuales, condiciones situacionales o contexto ambiental del estudiante de secundaría en el que son expuestos al uso y abuso de drogas.

Los resultados de los FR frente al consumo de alcohol que se identificaron fueron consistentes con lo pronunciado por Catalano, Hawkins, et al., (1996); Hawkins, Catalano y Miller, (1992)(24-25), quienes afirman la relevancia a la disminución de los $\mathrm{FR}$, pues un amplio conjunto de ellos hacen que se incremente la probabilidad de que la persona consuma drogas. Refieren también, que los problemas emocionales entre los jóvenes incrementan el riesgo de que incurran en el consumo de alcohol.

Los resultados del FR relaciones familiares es vulnerable, son congruentes con la recomendación del CONADIC (2008) y INSP, (2008)(18,21), que afirma que, los adolescentes tienen más probabilidad consumir alcohol cuando han sido expuestos a la oportunidad y de progresar hacia la dependencia cuando han usado alcohol. EI FR relaciones con amigos es vulnerable con más del $25 \%$, se tiene que cerrar la brecha en estos FR para ser consistentes con el CONADIC (2008) y INSP, (2008) $)^{(18,21)}$, que afirma que las generaciones actuales presentan mayor accesibilidad al alcohol, mayor consumo y mayor probabilidad de progresar del abuso a la dependencia que las generaciones anteriores.

Los resultados del FR nivel educativo son consistentes con CONADIC (2008) y INSP, (2008) (18,21), cuando afirma que los problemas emocionales entre los jóvenes incrementan el riesgo de que incurran en el consumo de alcohol.

Los resultados del FR interés laboral, son congruentes con el CONADIC (2008) y INSP $(2008)^{(18,21)}$, y con lo pronunciado por Catalano, Hawkins, et al. (1996); Hawkins, Catalano y Miller $(1992)^{(24-25)}$. Se debe dar gran relevancia a la disminución de estos FR, pues un amplio conjunto de ellos hacen que se incremente la probabilidad de que los adolescentes consuman alcohol.

La relación y efecto de los factores de riesgo sobre el consumo de alcohol, son diferentes a lo encontrado por la CONADIC (2008) y el INSP $(2008)^{(18,21)}$. Se encuentran efectos de los factores personales, sexo y edad y FR sobre el consumo de alcohol. La edad como predictora es consistente con Peréz et. al. $(2007)^{(7)}$, pero no con el género como factor personal biológico según Pender (2006) ${ }^{(13)}$.

\section{Conclusiones}

Los datos indicam que la población de adolescentes en el presente estúdio aunque muestre un consumo local menor ou igual al consumo nacional, sugiere que ese grupo de estudiantes está vulnerable a factores de riesgo tanto de origen personal como interpersonal. Esta evidencia la necesidad de implementar acciones preventivas locales.

Como limitaciones del estudio se pueden indicar el muestreo de tipo aleatorio y la utilización de instrumentos de recolección de datos de utilización restricta.

\section{Agradecimientos}

A la Secretaría Nacional Antidrogas (SENAD) de Brasil, a la Escuela de Enfermería de Ribeirao Preto de la Universidad de Sao Paulo y a la Dra. Margarita Antonia Villar Luis, por el apoyo brindado en la capacitación y formación de profesionales de la salud Latinoamericanos en la investigación de Adicciones.

\section{Referencias}

1. Naciones Unidas Contra las Drogas y el Delito (UNODC). Informe Mundial sobre las Drogas 2012. Nova lorque: Organización Mundial de la Salud; 2012.

2. Organización Panaméricana de la Salud [Internet]. Alcohol y atención primaria de la salud: informaciones clínicas básicas para la identificación y el manejo de riesgos y problemas. Washington, D.C.; 2008. [Acceso 13 enero 2010]. Disponible en: http://www.paho.org/ Spanish/DD/PUB/Alcohol_Aten_prim_web.pdf

3. Organización Mundial de la Salud [Internet]. Datos y cifras de alcohol. Nota descriptiva 349. Washington, D.C.; 2011. [Acceso 13 enero 2011]. Disponible en: http://www. who.int/mediacentre/factsheets/fs349/es/index.html

4. Ariza C, Nebot M, Villalbí JR, Díez E, Tomás Z, Valmayor $S$. Tendencias en el consumo de tabaco, alcohol y cannabis de los escolares de Barcelona (19871999). Gac Sanit. 2003;17: 190-5.

5. Comisión Nacional contra las Adicciones [Internet]. Palabras del Dr. Carlos Tena Tamayo, Comisionado Nacional contra las Adicciones. Conadic; 2011. [Acceso 15 nov 2011]. Disponible en: http://www.conadic.salud.gob. mx/prensa/boletines2011/palabrasavancesNV_300311. html

6. Ministerio de Sanidad y Consumo (ES) [Internet]. Encuesta Nacional de Salud de 2006. Madrid; 2007. 
7. Pérez MA, Leal HFJ, Jiménez PI, Mesa GI, Martínez FML, Pérez M R. Evolución del consumo de sustancias tóxicas en los adolescentes de una zona urbana. Atención Primaria. 2007;39(6):299-304.

8. Herrera-Vazquez M, Wagner F, Velazco Mondragón E, Borges G, LazcanoPonce E. Onset of alcohol and tobacco use and transition to other drug use among students from Morelos, Mexico. Salud Pública Mex. 2004;46:132-40.

9. Organización Mundial de la Salud [Internet]. Programación para la Salud y el Desarrollo de los Adolescentes. Ginebra: OMS; 1999. Serie de Informes Técnicos, 886. [Acceso 13 enero 2011]. Disponible en: http://www.paho.org

10. Agencia Mexicana de Noticias (Internet). Resultados preliminares de la encuesta nacional de adicciones. 2008. [Acceso 19 junio 2010]. Disponible en: http:// www.agenciamn.com/index.php/Salud/Se-presentanlos-resultados-preliminares-de-la-encuesta-nacional-deadicciones-2008.html

11. Secretaría de Salud (MX). Programa Nacional de Salud 2007-2012. Por un México sano: construyendo alianzas para una mejor salud. México, D.F.: Secretaría de Salud; 2007.

12. Oliva A, Parra A, Sánchez-Queija I, López F. Estilos educativos materno y paterno: evaluación y relación con el ajuste adolescente. Anales Psicol. 2007; 23:1-10.

13. Pender NJ, Murdaugh CL, Parsons M A. Health promotion in nursing practice. 4.ed. Ed. New Jersey: Prentice Hall; 2006. 397 p.

14. Marino MC, González-Fortaleza C, Andrade PE, Medina- Mora ME. Validación de un cuestionario para detectar adolescentes con problemas por el uso de drogas. Salud Mental. 1998;21:21-36.

15. Molinero O, Salguero A, Castro-Piñero J, Mora J, Márquez S. Substance abuse and health self-perception in Spanish children and adolescents. Nutr Hosp. [Internet]. 2011 [Acceso 19 nov 2012]. Apr;26(2):402-9. Disponible en: http://scielo.isciii.es/scielo.php?script=sci_ arttext\&pid=S0212-16112011000200024\&Ing=en.

16. Gómez A, et. al. Consumo de drogas lícitas e ilícitas por estudantes universitários. Revista de la Facultad de Ciencias de la Salud, 2007; 11(3), 41-45.

17. Orgaz Gallego MP, Segovia Jiménez M, Lopez de Castro F, Tricio Armero MA. Consumo de alcohol en escolares toledanos: motivos y alternativas. Aten Primaria. 2005 Oct; 36(6): 297-302.

18. Consejo Nacional Contra las Adicciones de la Secretaría de Salud Pública. Encuesta Nacional de Adicciones. Cuernavaca Morelos, Instituto Nacional de Salud Pública; 2007.

19. Plan Nacional sobre Drogas (ES). Comisión Nacional de Investigación en Drogas. Madrid: Ministerio del
Interior; 2001.

20. Consejo Nacional Contra las Adicciones de la Secretaría de Salud Publica. Encuesta Nacional de Adicciones 2008. Cuernavaca Morelos, Instituto Nacional de Salud Pública (INSP); 2008.

21. Anicama J. Impacto de los factores de riesgo y factores protectores en el desarrollo de la conducta adictiva. En: Zavaleta A, editores. Factores de riesgo y protección en el consumo de drogas en la juventud. Lima: CEDRO; 2002. p. 97-129.

22. Clayton RR. Transitions in drug use: Risk and protective factors. En: Glantz M, Pickens R, editores. Vulnerability to drug abuse. Washington, DC: American Psychological Association; 1992. p. 15-51.

23. Nazar B, Tapia R, Villa A, León G, Medina-Mora M, Salvatierra B. Factores asociados al consumo de drogas em adolescentes de áreas urbanas de México. Salud Publica de México. 1994;36(8):646-54.

24. Hawkins JD, Catalano RF, Miller JL. Risk and protective factors for alcohol and other drug problems in adolescence and early adulthood. Implications for substance abuse prevention. Psychol Bull. 1992;112(2):64-105.

25. Catalano FR, Hawkins JD. A social development model: A theory of antisocial behavior. In: Hawkings JD. Delinquency and crime: Current theories. Cambridge: Cambridge Press; 1996. p. 149-197. 